\title{
Crescimento e capacidade fotossintética da cultivar de amendoim BR 1 sob condições de salinidade
}

\author{
Erika S. A. G raciano ${ }^{1}$, Rejane J. M. C. N ogueira², D anúbia R. M. Lima ${ }^{3}$, \\ Cinthya M. Pacheco ${ }^{4} \&$ Roseane C. Santos ${ }^{5}$
}

\begin{abstract}
RESU MO
Objetivou-se com este trabalho avaliar o crescimento e a capacidade fotossintética da cultivar de amendoim BR 1, submetida a salinidade. 0 experimento foi conduzido em casa de vegetação, em delineamento inteiramente casualizado com quatro níveis de salinidade da solução, expressos em condutividade elétrica $\left(1,0 ; 3,5 ; 6,0\right.$ e $\left.8,5 \mathrm{dS} \mathrm{m}^{-1}\right)$ e seis repetições. A cultivar BR 1 apresentou, quando cultivada sob estresse salino, mecanismos fisiológicos capazes de assegurar seu crescimento. 0 crescimento do sistema radicular e a estabilidade dos pigmentos fotossintéticos foram as variáveis maìs relacionadas com a tolerância à salinidade. Contudo, a cultivar BR 1 é sensível à salinidade quando a condutividade elétrica é de $8,5 \mathrm{dS} \mathrm{\textrm {m } ^ { - 1 }}$, afetando a condutância estomática e a assimilação fotossintética de $\mathrm{CO}_{2}$. A BR 1 apresenta resistência a ambientes salinos.
\end{abstract}

Palavras-chave: Arachis hypogaea, estresse salino, crescimento, fotossíntese

\section{Growth and photosynthetic capacity of the peanut cultivar BR 1 under saline conditions}

\begin{abstract}
The objective of this study was to evaluate the growth and photosynthetic capacity of BR 1 peanut cultivar submitted to salinity. The experiment was conducted in greenhouse, using a randomized design, with four levels of salinity, expressed in electrical conductivity of solution (1.0, 3.5, 6.0 and $\left.8.5 \mathrm{dS} \mathrm{m}^{-1}\right)$, with six replications. BR 1 showed physiological mechanisms capable of ensuring their growth in environments prone to salinity. The grow th of root system and stability of photosynthetic pigments were the variables related to tolerance to salinity. However, the cultivar is sensitive to salinity when the electrical conductivity is $8.5 \mathrm{dS} \mathrm{m}^{-1}$, affecting the stomatal conductance and photosynthetic assimilation of $\mathrm{CO}_{2}$. BR1 showed resistance to saline environments.
\end{abstract}

Key words: Arachis hypogaea, saline stress, growing, photosynthesis

Trabalho submetido e selecionado no primeiro Simpósio Brasileiro de Salinidade realizado de 12-15/10/2010 em Fortaleza, Ceará, Brasil ${ }_{1}^{1}$ Programa de Pós-G raduação em Ciência do Solo/U FRPE. Rua Manuel de Medeiros, s/n, CEP 52171-900, Recife, PE. Fone: (81) 3320-6220. E-mail: erikagraciano@yahoo.com.br

2 Departamento de Biologia/UFRPE. Fone: (81) 3320 6352. E-mail: rjmansur1@gmail.com

3 Programa de Pós-Graduação em Ciência do Solo/U FRPE. Fone: (81) 3320-6220. E-mail: danubiaramosm@hotmail.com

${ }^{4}$ Programa dePós-Graduação em G enética/UFPE. Av. M oraes Rego, 1235, CEP 50670-901, Recife, PE. Fone: (81)2126-8512. E-mail: cinthya.pacheco@hotmail.com ${ }^{5}$ Embrapa Algodão. Rua O swaldo Cruz, 1143, CEP 58107-720, Campina G rande, PB. Fone: (83) 3341-3608; 3322-7751. E-mail: caval@cnpa.embrapa.br 


\section{INTRODUÇÃO}

O amendoim (Arachis hypogaea L.) é uma oleaginosa de grande importância econômica, cultivada mundialmente em países desenvolvidos e em desenvolvimento, com uma produção de 31 milhões de toneladas de grãos (USDA, 2010), para atender aos mercados de alimento e oleoquímico. No Brasil, a produção do amendoim se situa em 300.000 toneladas, obtidas nas regiões Sudeste, Sul, Centro-Oeste e Nordeste (IBGE, 2010). Nesta última região a lavoura do amendoim é uma atividade de grande interesse por parte de pequenos e médios agricultores que vivem da agricultura familiar.

No aspecto climático, contudo, a elevada evapotranspiração potencial da região Nordeste (Silva \& Amaral, 2007), associada ao manejo inadequado do solo e da água de irrigação, tem ocasionado elevações expressivas nos níveis de salinidade, afetando a produtividade da lavoura. De acordo com Silva et al. (2008), cerca de 23.000 ha dos perímetros irrigados na região Nordeste estão afetados por sais. Nas regiões afetadas por sais a salinidade é considerada um dos principais estresses abióticos que afetam a produtividade e a qualidade das culturas (Gondim et al., 2010), induzindo redução do crescimento das plantas (Garcia et al., 2010; Oliveira et al., 2010), além do abandono de lotes de terra nos perímetros irrigados da região Nordeste brasileira (Lacerda et al., 2009).

A resposta das plantas sob diferentes condições de salinidade é um fenômeno extremamente complexo, envolvendo alterações morfológicas e de crescimento, além de processos fisiológicos e bioquímicos. Deste modo, a sobrevivência das plantas em condições de salinidade pode exigir processos adaptativos envolvendo a absorção, o transporte e a distribuição de íons nos vários órgãos da planta, bem como a compartimentação de nutrientes minerais dentro das células (Zanandres et al., 2006; Garcia et al., 2010). As alterações no metabolismo induzidas pela salinidade são resultado de várias respostas fisiológicas da planta, dentre as quais se destacam as modificações no crescimento, comportamento estomático e capacidade fotossintética.

O declínio da produtividade das culturas em condições de salinidade elevada, mediante a redução do crescimento das plantas, é atribuído principalmente à baixa capacidade fotossintética, devido a limitações estomáticas e não estomáticas (Silva et al., 2008), decorrentes do fechamento estomático e da diminuição na atividade de fixação do carbono (Zanandres et al., 2006). A redução da capacidade fotossintética em função da salinidade pode ser também atribuída à diminuição do conteúdo dos pigmentos fotossintéticos. A salinidade reduz o teor de clorofila em plantas sensíveis a salinidade e aumenta em plantas tolerantes ao sal (Jamil et al., 2007).

Os processos de crescimento são particularmente sensíveis ao efeito da salinidade, de modo que a taxa de crescimento e a capacidade fotossintética, são bons parâmetros para a avaliação dos efeitos da salinidade, tal como a capacidade da planta para tolerar esse estresse (Correia et al., 2009; Garcia et al., 2010). Alguns estudos demonstram os efeitos deletérios da salinidade sobre o crescimento do amendoim, com base em variáveis diretamente relacionadas com o desenvolvimento vegetal
(Correia et al., 2005; Correia et al., 2009). No entanto, os prejuízos causados pelo estresse salino dependem de sua duração, sua severidade e do estádio de desenvolvimento da planta (Garcia et al., 2010).

De acordo com Benincasa (2003), a análise de crescimento é um meio prático e preciso para se avaliar o crescimento e inferir a contribuição de diferentes processos fisiológicos sobre o comportamento vegetal. Este método pode ser de grande valor na avaliação de diferenças intra e interespecíficas das diversas características que definem a capacidade produtiva da planta (Azevedo Neto \& Tabosa, 2000).

A planta do amendoim, especialmente da subespécie $A$. hypogaea fastigiata, possui ampla capacidade de adaptação a ambientes de clima semiárido (Santos et al., 2005; ; Araújo et al., 2008; Azevedo Neto et al., 2010). Além disso, esta espécie apresenta variabilidade de natureza varietal com relação a tolerância ao estresse salino (Correia et al., 2005; Nogueira \& Távora, 2005). A Embrapa Algodão dispõe de três cultivares de amendoim fastigiata, denominadas BR 1, BRS 151 L7 e BRS Havana, todas de alta produtividade e tolerantes ao clima semiárido nordestino (Santos et al., 2005).

Em estudos desenvolvidos sobre as relações hídricas e o ajustamento osmótico com essas cultivares, foi demonstrado que elas possuem elevada tolerância a condições simuladas de estresse hídrico (Nogueira \& Santos, 2000; Correia \& Nogueira, 2004). No aspecto da resistência a salinidade, contudo, as informações são escassas, especialmente para a cultivar BR 1 que é a de maior difusão na região Nordeste (Santos et al., 2005). Considerando-se a importância da lavoura do amendoim no agronegócio regional e, ainda, a expansão da cultura nas áreas irrigadas do Vale do São Francisco, desenvolveu-se o presente trabalho que teve como objetivo investigar o crescimento e a capacidade fotossintética da cultivar BR1 submetida a diferentes níveis de salinidade.

\section{Material E MÉTODOS}

O experimento foi conduzido em casa de vegetação do Laboratório de Fisiologia Vegetal do Departamento de Biologia da Universidade Federal Rural de Pernambuco, em Recife, PE, Brasil. As sementes de amendoim (Arachis hypogaea L.), cultivar BR 1, foram fornecidas pela Embrapa Algodão, Campina Grande, PB.

As sementes foram semeadas em bandejas de polietileno contendo um substrato composto por uma mistura de solo, pó de coco e esterco animal, na proporção de 1:1:1 (v/v/v). Após a germinação e a emissão do primeiro par de folhas definitivas, as plântulas foram transferidas para vasos de polietileno, contendo $12 \mathrm{~kg}$ de areia previamente lavada, onde foram mantidas durante três dias para aclimatação. Os tratamentos salinos foram aplicados nas plântulas sete dias após a germinação, pela adição de concentrações crescentes de $\mathrm{NaCl}$ $(0,25,50$ e $75 \mathrm{mM})$ em solução nutritiva de Hoagland \& Arnon (1950), diluída em $1 / 2$, os quais foram expressos pela condutividade elétrica (CE) da solução: 1,0; 3,5; 6,0 e 8,5 dS m ${ }^{-1}$, respectivamente. As plantas foram irrigadas com a solução nutritiva $1 / 2$ a cada dois dias intercalando com água destilada. 
O delineamento experimental foi o inteiramente casualizado com quatro tratamentos salinos com $\mathrm{NaCl}(0,25,50$ e $75 \mathrm{mM})$ correspondendo aos valores de CE de 1,0; 3,5; 6,0 e 8,5 dS m ${ }^{-1}$. A parcela experimental foi constituída por um vaso contendo uma planta e todos os tratamentos foram representados por seis repetições $(n=6)$.

Para manter os referidos níveis de salinidade no substrato, realizaram-se as leituras da CE, semanalmente, com o auxílio do condutivímetro marca Schott, modelo LF 613T. Durante o período experimental as amplitudes de temperatura e da umidade relativa do ar, no interior da casa de vegetação, variaram de 24,3 a $31^{\circ} \mathrm{C}$ e de 50,2 a $75 \%$, respectivamente.

As avaliações de crescimento foram realizadas durante todo o período experimental. As medidas de altura, número de folhas e diâmetro da haste principal, foram mensurados a cada sete dias. A altura foi medida com auxílio de uma trena a partir da base da haste principal até a última gema do meristema apical. Para avaliação do número de folhas foram consideradas as folhas com limbo expandido. Na avaliação do diâmetro da haste principal utilizou-se um paquímetro digital de marca Calipers, com precisão de $0,002 \mathrm{~mm}$, a $1 \mathrm{~cm}$ da base da haste.

Para análise da matéria seca foram coletadas as folhas (MSF), hastes (MSH), raízes (MSR) e planta (MST) de cada tratamento, aos 52 dias de diferenciação dos tratamentos salinos, sendo secadas a $65{ }^{\circ} \mathrm{C}$ em estufa de circulação forçada. Com esses dados calculou-se a alocação de biomassa para as folhas (ABF), hastes $(A B H)$, raízes $(A B R)$ e a relação raiz/parte aérea $(R / P a)$, segundo Benincasa (2003).

As avaliações da condutância estomática $\left(\mathrm{gs}, \mathrm{mol} \mathrm{m}^{-2} \mathrm{~s}^{-1}\right)$, transpiração foliar $\left(\mathrm{E}, \mu \mathrm{mol} \mathrm{m} \mathrm{m}^{-2} \mathrm{~s}^{-1}\right)$ e fotossíntese $\left(\mathrm{A}, \mu \mathrm{mol} \mathrm{m} \mathrm{m}^{-2} \mathrm{~s}^{-1}\right)$ foram realizadas aos 52 dias após diferenciação dos níveis salinos, às 11:00 h, por meio do sistema portátil de medições de trocas gasosas, modelo LI-6200 (LI-COR, Lincoln, NE, USA).

$\mathrm{O}$ déficit de pressão de vapor entre a folha e o ar ( $\left.\mathrm{DPV}_{\text {folha-ar }}\right)$ variou entre 1,42 a 2,22 kPa durante o horário de avaliação (11:00 h), cujos valores de DPV $_{\text {folha-ar }}$ são considerados baixos por Erismann et al. (2006).
A estimativa do conteúdo de clorofila na folha (SPADF) e planta (SPADP) foi determinada por meio dos valores do índice de SPAD-502, utilizando-se o medidor portátil de clorofila SPAD-502 (Minolta Camera Co. Ltd.). Para determinação do SPADF utilizaram-se os quatro folíolos de cada folha e se realizaram oito leituras na folha, das quais duas leituras em cada folíolo, calculando-se a média para a folha amostrada no próprio medidor; para determinação do SPADP foram feitas 24 leituras aleatórias; após o término das leituras foram coletadas amostras das folhas utilizadas na determinação da SPADF, para determinação dos pigmentos fotossintéticos.

Juntamente com a estimativa do conteúdo de clorofila pelo índice de SPAD-502, realizou-se a determinação dos teores das clorofilas a, b e carotenóides mantendo-se, por 24 h, $100 \mathrm{mg}$ de tecido foliar fresco em tubos de ensaio hermeticamente fechados e protegidos da luz, contendo $10 \mathrm{~mL}$ de acetona a $80 \%$. A determinação foi realizada utilizando-se um espectrofotômetro Biospectro, modelo SP-220, nos comprimentos de onda de 663, 645 e $470 \mathrm{~nm}$, respectivamente, de acordo com as fórmulas descritas por Lichtenthaler (1987).

Os dados obtidos foram submetidos à análise de variância e as médias comparadas pelo teste de Tukey a nível de 5\% probabilidade, utilizando-se o Proc Mixed do SAS (SAS Inst. Inc., 1996). O número de folhas foi transformado em $\sqrt{\mathrm{x}+0,5}$.

\section{RESULTADOS E DISCUSSÃO}

Os diferentes níveis de salinidade avaliados neste trabalho influenciaram significativamente $(\mathrm{p}<0,05)$ a altura da planta, número de folhas e diâmetro da haste principal da cultivar BR 1; os resultados dessas variáveis estão apresentados na Tabela 1. A altura da planta e o número de folhas apresentaram diminuição a partir do $15^{\circ}$ dia após a aplicação dos tratamentos salinos, com redução de 62,4 e 33,6\%, respectivamente, quando submetidas aos níveis de $8,5 \mathrm{dS} \mathrm{m}^{-1}$ de CE em relação ao controle $\left(1,0 \mathrm{dS} \mathrm{m}^{-1}\right)$. A redução dessas variáveis se manteve ao longo

Tabela 1. Altura da planta, número de fol ha e diâmetro da haste da cultivar BR 1 sob diferentes níveis de salinidade em casa de vegetação ${ }^{1}$

\begin{tabular}{|c|c|c|c|c|c|c|c|c|}
\hline \multirow{2}{*}{$\begin{array}{l}\text { Níveis de CE* } \\
\left.\text { (dS } \mathrm{m}^{-1}\right)\end{array}$} & \multicolumn{8}{|c|}{ Número de dias após a aplicação dos tratamentos salinos } \\
\hline & 1 & 8 & 15 & 22 & 29 & 38 & 45 & 52 \\
\hline & \multicolumn{8}{|c|}{ Altura da planta (cm) } \\
\hline 1,0 & $1,10 \mathrm{a}$ & 1,98 a & 5,76 a & $14,20 \mathrm{a}$ & 24,70 a & 32,68 a & 33,98 a & 39,58 a \\
\hline 3,5 & $1,08 \mathrm{a}$ & $1,40 \mathrm{a}$ & $3,86 a b$ & $8,54 b$ & $18,56 \mathrm{~b}$ & $23,74 \mathrm{~b}$ & $25,44 b$ & $32,78 \mathrm{~b}$ \\
\hline 6,0 & $1,12 \mathrm{a}$ & 1,60 a & $4,06 a b$ & $6,02 b$ & $13,06 \mathrm{c}$ & $18,10 \mathrm{C}$ & $18,96 \mathrm{c}$ & $26,18 \mathrm{c}$ \\
\hline \multirow[t]{2}{*}{8,5} & $0,86 \mathrm{a}$ & $1,58 \mathrm{a}$ & $2,14 \mathrm{~b}$ & $4,90 \mathrm{~b}$ & $9,18 \mathrm{c}$ & $13,14 \mathrm{C}$ & $14,96 \mathrm{C}$ & $23,14 \mathrm{c}$ \\
\hline & \multicolumn{8}{|c|}{ Número de folhas** } \\
\hline 1,0 & $3,00 a$ & 8,80 a & $20,20 \mathrm{ab}$ & $33,60 a$ & $38,4 a b$ & 59,00 a & 69,80 a & 88,40 a \\
\hline 3,5 & $3,00 \mathrm{a}$ & 8,40 a & $20,80 a$ & $33,40 a$ & $44,0 \mathrm{a}$ & $57,60 a b$ & $70,00 \mathrm{a}$ & $87,20 a b$ \\
\hline 6,0 & $3,00 a$ & 9,00 a & $14,80 \mathrm{bc}$ & $26,80 \mathrm{~b}$ & $36,8 \mathrm{ab}$ & $50,80 a b$ & $56,60 \mathrm{~b}$ & $71,80 \mathrm{c}$ \\
\hline \multirow[t]{2}{*}{8,5} & $2,60 \mathrm{a}$ & $6,60 \mathrm{a}$ & $13,40 \mathrm{c}$ & $23,80 \mathrm{~b}$ & $32,4 \mathrm{~b}$ & $48,60 \mathrm{~b}$ & $56,60 \mathrm{~b}$ & $75,00 \mathrm{c}$ \\
\hline & \multicolumn{8}{|c|}{ Diâmetro da haste (mm) } \\
\hline 1,0 & $3,10 \mathrm{a}$ & $3,57 \mathrm{a}$ & $3,84 a$ & $4,10 a$ & 4,89 a & $5,99 a$ & $6,27 a$ & $6,62 b$ \\
\hline 3,5 & $3,00 \mathrm{a}$ & 3,29 a & 3,69 a & 3,95 a & $4,76 \mathrm{a}$ & 6,21 a & $6,68 a$ & 7,91 a \\
\hline 6,0 & $2,90 \mathrm{a}$ & 3,48 a & 3,98 a & $4,26 a$ & $4,55 \mathrm{a}$ & $6,27 \mathrm{a}$ & $7,10 \mathrm{a}$ & 7,55 a \\
\hline 8,5 & $2,80 \mathrm{a}$ & $3,37 \mathrm{a}$ & $3,53 \mathrm{a}$ & $3,96 \mathrm{a}$ & $4,26 \mathrm{a}$ & $6,35 \mathrm{a}$ & $6,80 a$ & $7,38 a b$ \\
\hline
\end{tabular}

${ }^{1}$ Médias seguidas de mesma letra nas colunas não diferem estatisticamente entre si a nível de $5 \%$ de probabilidade pelo teste de Tukey.

* CE - Condutividade elétrica da solução.

** Dados transformado em $\sqrt{\mathrm{x}+0,5}$ 
do período experimental (22, 29, 38, 45 e 52 dias após a aplicação dos tratamentos salinos); aos 52 dias, a altura da planta reduziu 33,8 e $41,5 \%$ e o número de folhas 18,7 e $15,1 \%$, quando submetidas a CE de 6,0 e $8,5 \mathrm{dS} \mathrm{m}^{-1}$, respectivamente, em relação ao controle. O diâmetro da haste só apresentou efeito significativo aos 52 dias após a aplicação dos tratamentos salinos, quando as plantas submetidas aos níveis de CE de 3,5 e $6,0 \mathrm{dS} \mathrm{m} \mathrm{m}^{-1}$ aumentaram significativamente em 19,5 e 14,0\% o diâmetro da haste, respectivamente, em relação ao controle.

Avaliando os efeitos do uso de águas de diferentes salinidades no crescimento das cultivares BR 1 e BRS 151 L7, Correia et al. (2005), constataram redução na altura da planta e no número de folhas aos 45 e 105 após a semeadura com o aumento da salinidade, sendo o crescimento da cv. BR 1 mais afetado aos 105 dias. Correia et al. (2009), analisando as características de crescimento de cultivares de amendoim (BR 1 e BRS 151 L7) sob diferentes condições de salinidade (condutividade elétrica da água de irrigação: 0,$4 ; 1,5 ; 3,0 ; 4,5$ e $6,0 \mathrm{dS} \mathrm{m}^{-1}$ ), verificaram decréscimo acentuado no número médio de folhas de $41 \%$ por aumento unitário da condutividade elétrica da água.

O decréscimo da altura da planta e do número de folhas da cultivar BR 1 verificado neste trabalho, pode estar relacionado à redução do potencial osmótico da solução do solo devido ao incremento dos níveis de salinidade. Segundo Garcia et al. (2010), com o incremento dos níveis de salinidade do solo possivelmente há redução do potencial matricial do solo, podendo causar resistência à absorção de água pelas plantas. Os mesmos autores relatam, também, que o aumento de pressão osmótica no solo pode atingir valores em que as plantas não terão forças de sucção suficiente para superar o potencial do solo. A consequência deste aumento da salinidade na solução do solo é a diminuição da disponibilidade e da absorção de água, afetando a divisão e o alongamento das células (Tester \& Davenport, 2003) diminuindo, assim, a altura da planta e o número de folhas.

A matéria seca da folha (MSF), haste (MSH) e total (MST) da planta aos 52 dias após a aplicação dos tratamentos salinos, não apresentou alteração significativa com o incremento dos níveis de salinidade (Tabela 2). A matéria seca da raiz (MSR) apresentou um aumento significativo de 84, 60 e 58\% nas plantas submetidas aos tratamentos de 3,5, 6,0 e 8,5 dS m ${ }^{-1} \mathrm{de}$ $\mathrm{CE}$, respectivamente, em relação ao controle $\left(\mathrm{CE}\right.$ de $\left.1,0 \mathrm{dS} \mathrm{m}^{-1}\right)$. Este incremento na MSR indica maior investimento da cultivar BR 1 em raiz, promovendo uma distribuição melhor do sistema

Tabela 2. M atéria seca da folha (MSF), haste (M SH ), raiz (MSR) e total (MST) da cultivar BR 1 sob diferentes níveis de salinidade em casa de vegetação ${ }^{1}$

\begin{tabular}{ccccc}
\hline Níveis de CE* & MSF & MSH & MSR & MST \\
\cline { 2 - 5 } (dS $^{-1}$ ) & \multicolumn{4}{c}{ (g planta $^{-1}$ ) } \\
1,0 & $6,72 \mathrm{a}$ & $10,99 \mathrm{a}$ & $2,19 \mathrm{~b}$ & $19,90 \mathrm{a}$ \\
3,5 & $6,57 \mathrm{a}$ & $12,04 \mathrm{a}$ & $4,02 \mathrm{a}$ & $22,64 \mathrm{a}$ \\
6,0 & $6,43 \mathrm{a}$ & $11,56 \mathrm{a}$ & $3,51 \mathrm{a}$ & $21,51 \mathrm{a}$ \\
8,5 & $6,13 \mathrm{a}$ & $9,99 \mathrm{a}$ & $3,47 \mathrm{a}$ & $19,60 \mathrm{a}$ \\
\hline CV (\%) & 13,19 & 12,16 & 21,25 & 11,16 \\
\hline
\end{tabular}

${ }_{1}^{1}$ Médias seguidas de mesma letra nas colunas não diferem entre si a nível de $5 \%$ de probabilidade pelo teste de Tukey.

* CE - Condutividade elétrica da solução radicular e permitindo maior exploração de área do solo, podendo resultar em, também, maior eficiência na absorção de água e nutrientes promovendo, então, melhores condições para seu crescimento sob condições salinas.

Correia et al. (2005), estudando o crescimento de duas cultivares de amendoim (BR1 e L7) irrigadas com águas de diferentes níveis de condutividade elétrica $(0,4 ; 1,5 ; 3,0 ; 4,5$ e $6,0 \mathrm{dS} \mathrm{m}^{-1}$ ) verificaram, aos 45 dias após semeadura, redução progressiva na fitomassa de folha e caule, com decréscimo de 2,66\% para a MSF e 3,85\% para MSC com o aumento unitário da CE na cultivar BR 1. Esses autores constataram inibição do crescimento radicular com a salinidade, aos 105 dias após semeadura, com um percentual de decréscimo por aumento unitário da CE de 8,2\% da MSR para a cultivar BR 1. Esses resultados divergem dos apresentados neste trabalho; entretanto, deve-se considerar a época de avaliação, uma vez que a cultivar BR 1 é de alta precocidade, apresentando ciclo de 89 dias após emergência (Santos et al., 2005).

Na Tabela 3 se acham os valores da alocação de biomassa das folhas $(\mathrm{ABF})$ e raízes $(\mathrm{ABR})$ e a relação raiz parte aérea $(\mathrm{R} /$ $\mathrm{Pa})$ da BR 1, cujas variáveis apresentaram efeito significativo com o incremento dos níveis de salinidade. A ABF apresentou redução de 14 e $11 \%$ nos níveis de 3,5 e $6,0 \mathrm{dS} \mathrm{m}^{-1}$ de $\mathrm{CE}$, respectivamente, em relação ao controle. Esta redução na $\mathrm{ABF}$ pode estar relacionada com a redução do número de folhas. A ABR aumentou significativamente 62, 51 e 63\% nos níveis de CE 3,5, 6,0 e 8,5 dS m m $^{-1}$, respectivamente, em relação ao controle, resposta que se mostra coerente com MSR e com a R/Pa. Por sua vez, a R/Pa apresentou aumento significativo de 75, 58 e $75 \%$ nos níveis 3,5, 6,0 e 8,5 dS m ${ }^{-1}$ de CE em relação ao controle, respectivamente. Embora se tenha constatado redução significativa da altura da planta neste trabalho, não se verificou alteração significativa na $\mathrm{ABH}$ com os níveis de salinidade impostos, o qual pode ser decorrente do aumento do diâmetro da haste da planta.

Tabela 3. Alocação de biomassa da folha (ABF), haste $(A B H)$, raiz $(A B R)$ e relação raiz parte aérea $(R / P A) d a$ cultivar BR 1 sob diferentes níveis de salinidade em casa de vegetação ${ }^{1}$

\begin{tabular}{ccccc}
\hline $\begin{array}{c}\text { Níveis de CE* } \\
\left.\text { (dS } \text { }^{-1}\right)\end{array}$ & ABF & ABH & ABR & R/Pa \\
\cline { 2 - 5 } 1,0 & $33,71 \mathrm{a}$ & $55,43 \mathrm{a}$ & $10,86 \mathrm{~b}$ & $12 \mathrm{~b}$ \\
3,5 & $29,10 \mathrm{~b}$ & $53,30 \mathrm{a}$ & $17,60 \mathrm{a}$ & $21 \mathrm{a}$ \\
6,0 & $29,90 \mathrm{~b}$ & $53,70 \mathrm{a}$ & $16,40 \mathrm{a}$ & $19 \mathrm{a}$ \\
8,5 & $31,31 \mathrm{ab}$ & $50,97 \mathrm{a}$ & $17,72 \mathrm{a}$ & $21 \mathrm{a}$ \\
\hline CV (\%) & 6,97 & 5,66 & 17,56 & 12,2 \\
\hline
\end{tabular}

${ }^{1}$ Médias seguidas de mesma letra nas colunas não diferem entre si a nível de $5 \%$ de probabilidade pelo teste de Tukey.

* CE - Condutividade elétrica da solução

Segundo Correia \& Nogueira (2004), a alocação de biomassa nos determinados órgãos da planta revela estratégia diferenciada à medida em que os níveis de estresse forem intensificados. Quando submetida a diferentes níveis de salinidade, a cultivar BR 1 alocou mais biomassa na raiz, favorecendo o desenvolvimento do sistema radicular. Embora Correia et al. (2005; 2009) tenham constatado sensibilidade da BR 1 à salinidade, foi possível, neste trabalho, constatar 
tolerância da mesma às condições de salinidade a que foi imposta, com base na baixa porcentagem de redução das variáveis de crescimento da parte aérea, exceto para a altura da planta, e no desenvolvimento do sistema radicular como estratégia de resistência à salinidade.

O comportamento estomático da cultivar BR 1 foi alterado com o incremento da salinidade, podendo ser examinado a partir da condutância estomática, transpiração foliar e assimilação fotossintética de $\mathrm{CO}_{2}$ na Figura 1. A condutância estomática (gs) da cv. BR 1 reduziu significativamente com o incremento da salinidade (Figura 1A). As plantas, quando submetidas às CE 3,5, 6,0 e 8,5 $\mathrm{dS} \mathrm{m}^{-1}$, apresentaram valores de gs de 1,61, 1,66 e $0,69 \mathrm{~mol} \mathrm{~m}^{-2} \mathrm{~s}^{-1}$, correspondendo a uma redução de 24,0, 21,7 e $67,4 \%$, respectivamente, em relação ao controle (CE de 1,0 dS $\mathrm{m}^{-1}$ ). A transpiração foliar (E) das plantas submetidas a CE de $3,5 \mathrm{dS} \mathrm{m}^{-1}$, aumentou significativamente, apresentado valor de $13,17 \mu \mathrm{mol} \mathrm{m}^{-2} \mathrm{~s}^{-1}$, correspondendo a um aumento de 5,36\% em relação ao controle; já as plantas submetidas a CE 8,5 dS m${ }^{-1}$
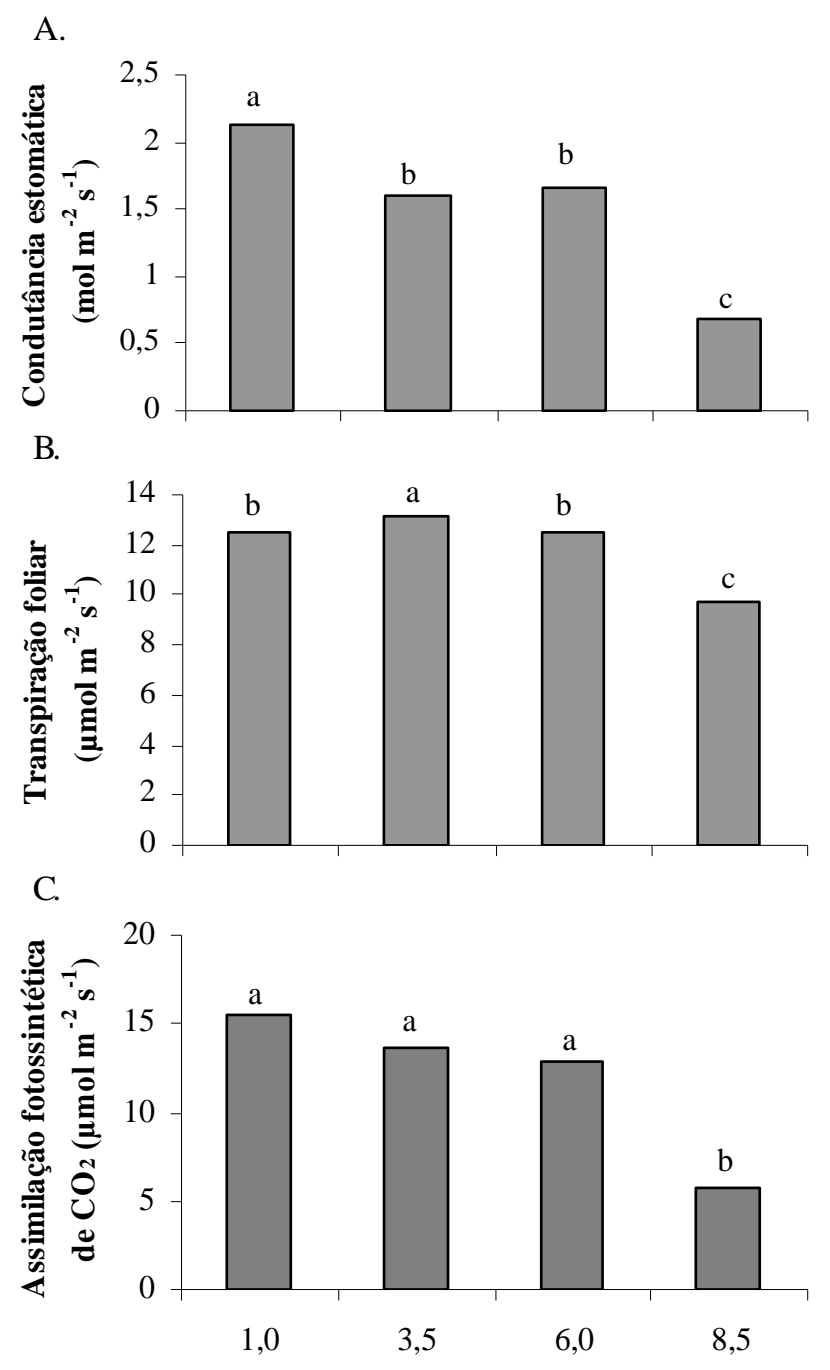

Condutividade elétrica $\left(\mathrm{dS} \mathrm{m}^{-1}\right)$

Obs.: Barras seguidas de mesma letra não diferem entre si a nível de $5 \%$ de probabilidade pelo teste de Tukey

Figura 1. Condutância estomática (A), transpiração foliar (B) e assimilação fotossintética de $\mathrm{CO}_{2}$ (C) de plantas de amendoim (cultivar BR 1) submetidas a diferentes níveis crescentes de salinidade apresentaram redução significativa da $\mathrm{E}$ de $4,71 \%$, em relação ao controle (Figura 1B). A assimilação fotossintética de $\mathrm{CO}_{2}$ (A) das plantas submetidas a CE 3,5 e 6,0 dS m $\mathrm{m}^{-1}$ apresentou valores de 13,7 e $12,9 \mu \mathrm{mol} \mathrm{m} \mathrm{m}^{-2} \mathrm{~s}^{-1}$, respectivamente, os quais não indicaram diferença significativa do controle $(15,46 \mu \mathrm{mol}$ $\left.\mathrm{m}^{-2} \mathrm{~s}^{-1}\right)$. As plantas submetidas a CE 8,5 dS m ${ }^{-1}$, mostraram valores de A de 5,8 $\mu \mathrm{mol} \mathrm{m} \mathrm{m}^{-2} \mathrm{~s}^{-1}$, correspondendo a uma redução significativa de $62,5 \%$ quanto ao controle (Figura 1C). Este valor de A, obtido nas plantas submetidas a $\mathrm{CE}$ de $8,5 \mathrm{dS} \mathrm{m}^{-1}$, se encontra abaixo do intervalo de 13 a $41 \mu \mathrm{mol} \mathrm{m}^{-2} \mathrm{~s}^{-1}$, relatados por Erismann et al. (2006) para plantas de amendoim.

O incremento nos níveis de salinidade causou redução da abertura estomática, confirmado pela redução dos valores da gs. Embora o fechamento estomático eleve a eficiência intrínseca no uso da água (Machado Filho et al., 2006), contribuiu para a redução nos valores de assimilação fotossintética de $\mathrm{CO}_{2}$ nas plantas submetidas a CE de $8,5 \mathrm{dS} \mathrm{m}^{-1}$, verificando-se dependência da $A$ com gs. Este resultado demonstra sensibilidade da cultivar BR 1 a esse nível de salinidade.

Os teores dos pigmentos fotossintéticos das plantas submetidas aos diferentes níveis de salinidade aumentaram significativamente (Tabela 4). A clorofila a apresentou um aumento da ordem de 53, 52 e 54\% nas plantas submetidas a CE de 3,5, 6,0 e 8,5 dS m ${ }^{-1}$, respectivamente, em relação ao controle, enquanto que na clorofila b esses aumentos foram de 36,57 e $55 \%$ nos mesmos tratamentos. Considerando a clorofila total da planta, os aumentos foram de 47, 48 e 54\%. Percebe-se que, na maior concentração de sal $\left(8,5 \mathrm{dS} \mathrm{m}^{-1}\right)$, os valores foram, praticamente, os mesmos para a clorofila a, b e total. O teor de carotenóides aumentou $31 \%$ nas plantas submetidas a CE de $3,5 \mathrm{dS} \mathrm{m}^{-1}$ e $21 \%$ nas submetidas a CE de $8,5 \mathrm{dS} \mathrm{m}^{-1}$.

Tabela 4. Teores de clorofilas $a, b$ e total em folhas de amendoim, cultivar BR 1, submetida a diferentes níveis de salinidade em casa de vegetação ${ }^{1}$

\begin{tabular}{|c|c|c|c|c|}
\hline \multirow{2}{*}{$\begin{array}{l}\text { Níveis de CE } \\
\text { (dS } \mathrm{m}^{-1} \text { ) }\end{array}$} & Clor $^{* * a}$ & Clor b & Clor Total & Carotenóides \\
\hline & \multicolumn{4}{|c|}{ (g planta $^{-1}$ ) } \\
\hline 1,0 & $1,26 b$ & $0,53 \mathrm{c}$ & $1,80 \mathrm{~b}$ & $0,19 \mathrm{c}$ \\
\hline 3,5 & $1,93 \mathrm{a}$ & $0,72 b$ & $2,65 a$ & $0,25 a$ \\
\hline 6,0 & $1,92 \mathrm{a}$ & $0,83 a$ & $2,67 a$ & $0,19 \mathrm{c}$ \\
\hline 8,5 & $1,94 \mathrm{a}$ & $0,82 a b$ & $2,77 \mathrm{a}$ & $0,23 b$ \\
\hline CV (\%) & 5,83 & 8,68 & 5,80 & 8,19 \\
\hline
\end{tabular}

${ }^{1}$ Médias seguidas de mesma letra nas colunas não diferem entre si ao nível de $5 \%$ de probabilidade pelo teste de Tukey

* CE - Condutividade elétrica da solução

** Clorofila

O conteúdo de clorofila na folha (SPADF) e na planta (SPADP), estimado pelo índice de SPAD-502 (Tabela 5), também apresentou aumento significativo com a salinidade. O SPADF apresentou aumento de 19,4\% nas plantas submetidas a CE 6,0 $\mathrm{dS} \mathrm{m} \mathrm{m}^{-1}$ e 19,7\% nas submetidas a CE de 8,5 dS m $\mathrm{m}^{-1}$. Para os níveis de CE 3,5, 6,0 e 8,5 dS m${ }^{-1}$, o SPADP teve aumento de $12,8,19,8$ e 18,5\% em relação ao controle, respectivamente. Este aumento verificado nos teores dos pigmentos fotossintéticos, evidencia resistência da cultivar BR 1 ao incremento da salinidade tendo como foco melhorar o desempenho da assimilação fotossintética de $\mathrm{CO}_{2}$ da planta para manter o seu crescimento e produção. 
Tabela 5. Conteúdo de clorofilas estimado pelo índice de SPAD em folhas de amendoim, cultivar BR 1, submetida a diferentes níveis de salinidade em casa de vegetação ${ }^{1}$

\begin{tabular}{ccc}
\hline Níveis de CE $^{*}\left(\mathbf{d S ~ ~ ^ { - 1 }}\right)^{-}$ & SPADF $^{*}$ & SPADP $^{* *}$ \\
1,0 & $35,70 \mathrm{~b}$ & $38,38 \mathrm{C}$ \\
3,5 & $41,23 \mathrm{ab}$ & $43,30 \mathrm{~b}$ \\
6,0 & $42,65 \mathrm{a}$ & $46,00 \mathrm{a}$ \\
8,5 & $42,75 \mathrm{a}$ & $45,50 \mathrm{ab}$ \\
\hline CV (\%) & 5,84 & 3,64 \\
\hline
\end{tabular}

${ }^{1}$ Médias seguidas de mes ma letra nas colunas não diferem entre si ao nível de $5 \%$ de probabilidade pelo teste de Tukey.

* Índice de SPAD-502 da folha

** Índice de SPAD-502 da planta

Analisando-se, de forma generalizada, os valores verificados nos teores dos pigmentos fotossintéticos e os dados de crescimento, constata-se que a cultivar de amendoim BR 1 apresenta resistência a salinidade.

\section{CONCLUSÕES}

1. Quando em condições de estresse salino, a cultivar BR 1 desenvolve mecanismos fisiológicos capazes de assegurar seu crescimento, fato considerado uma estratégia adaptativa a ambientes salinos; entre os mecanismos mais inerentes à resistência, o sistema radicular e os pigmentos fotossintéticos são os mais responsivos.

2. A cultivar BR 1 apresenta sensibilidade a salinidade quando submetida ao nível de condutividade elétrica de 8,5 $\mathrm{dS} \mathrm{m}^{-1}$, afetando a condutância estomática e a assimilação de $\mathrm{CO}_{2}$.

\section{AgRADECIMENTOS}

Os autores agradecem à CAPES, pela concessão da Bolsa de Mestrado em Botânica, ao primeiro autor. Ao mesmo tempo, agradecem a FACEPE, pela Bolsa de Iniciação Científica concedida ao terceiro autor.

\section{LITERATURA CITADA}

Araújo, A. C.; Beltrão, N. E. de M.; Morais, M. S.; Araújo, J. L. O.; Cunha, J. L. X. L.; Paixão, S. L. Indicadores agroeconômico na avaliação do consórcio algodão + amendoim. Ciência e Agrotecnologia, v.32, p.1467-1472, 2008.

Azevedo Neto, A. D.; Nogueira, R. J. M. C.; Melo Filho, P. A.; Santos, R. C. Physiological and biochemical responses of peanut genotypes to water déficit. Journal of Plant Interactions, v.5, p.1-10, 2010.

Azevedo Neto, A. D.; Tabosa, J. N. Estresse salino em plântulas de milho: Parte I análise de crescimento. Revista Brasileira de Engenharia Agrícola e Ambiental, v.4, p.159-164, 2000.

Benincasa, M. M. P. Análise de crescimento de plantas (noções básicas). Jaboticabal: FUNEP, 2003. 42p.
Correia, K. G.; Fernandes, P. D.; Gheyi, H. R.; Gurgel, M. T.; Rodrigues, L. N. Crescimento do amendoinzeiro irrigado com águas salinas. Revista Brasileira de Engenharia Agrícola e Ambiental, v.9, p.81-85, 2005.

Correia, K. G.; Fernandes, P. D.; Gheyi, H. R.; Nobre, R. G.; Santos, T. S. Crescimento, produção e características de fluorescência da clorofila a em amendoim sob condições de salinidade. Revista Ciência Agronômica, v.40, p.514-521, 2009.

Correia, K. G.; Nogueira, R. J. M. C. Avaliação do crescimento do amendoim (Arachis hypogaea L.) submetido a déficit hídrico. Revista de Biologia e Ciência da Terra, v.4, n.2, 2004.

Erismann, N. M.; Machado, E. C.; Godoy, I. J. Capacidade fotossintética de genótipos de amendoim em ambiente natural e controlado. Pesquisa Agropecuária Brasileira, v.41, p.1099-1108, 2006.

Garcia, G. O.; Nazário, A. A.; Moraes, W. B.; Gonçalves, I. Z.; Madalão, J. C. Respostas de genótipos de feijoeiro á salinidade. Engenharia na Agricultura, v.18, p. 330-338, 2010.

Gondim, F. A.; Gomes Filho, E.; Lacerda, C. F.; Prisco, J. T.; Azevedo Neto, A. D.; Marques, E. C. Pretreatment with $\mathrm{H}_{2} \mathrm{O}_{2}$ in maize seeds: Effects on germination and seedling acclimation to salt stress. Brazilian Journal of Plant Physiology, v.22, p.103-112, 2010.

Hoagland, D. R.; Arnon, D. I. The water-culture method for growing plants without soil. California: California Agricultural Experiment Station, 1950. 32p. (CAES, Circular 347).

IBGE. Instituto Brasileiro de Geografia e Estatística. Produção Agrícola. http://www.ibge.gov.br/home/estatistica/ indicadores/agropecuaria/lspa/lspa_201003_7.shtm. 09 Set. 2010.

Jamil, M.; Rehman, S.; Lec, K. J.; Kim, J. M.; Kim, H. S.; Rha, E. S. Salinity reduced growth PS2 photochemistry and chlorophyll content in radish. Scientia Agrícola, v.64, p.111118, 2007.

Lichtenthaler, H. K. Chlotophylls and carotenoids: Pigments of photosynthetic biomembranes. In: Packer, L.; Douce, R. (ed.). Methods in enzimology academic. London, 1987. p.350381.

Machado Filho, J. A.; Campostrini, E.; Yamanishi, O. K.; Fagundes, G. R. Efeito da umidade do ar nas trocas gasosas em folhas de mamoeiro (Carica papaya L.) cultivado em condições de campo. Bragantia, v.65, p.185-196, 2006.

Nogueira, R. J. M. C.; Santos, R. C. dos. Alterações fisiológicas no amendoim submetido ao estresse hídrico. Engenharia Agrícola, v.4, p.41-45, 2000.

Nogueira, R. J. M. C.; Távora, F. J. A. F. Ecofisiologia do amendoim (Arachis hipogaea L.). In: Santos, R. C. dos (ed.). $O$ agronegócio do amendoim no Brasil. Campina Grande: EMBRAPA, 2005. Cap.3, p.71-122.

Oliveira, I. R. S.; Oliveira, F. N.; Medeiros, M. A.; Torres, S. B.; Teixeira, F. J. V. Crescimento inicial do pinhão-manso (Jatropha curcas L.) em função da salinidade de água de irrigação. Caatinga, v.23, p.40-45, 2010.

Santos, R. C.; Godoy, J. I.; Fávero, A. P. Melhoramento do amendoim. In: Santos, R. C. dos. (ed.). O agronegócio do amendoim no Brasil. Campina Grande: EMBRAPA, 2005. Cap.4, p.123-192. 
SAS Inst. SAS statistics user's guide. Release version 6., Cary: SAS Inst. Inc. 1996.

Silva, E. C.; Nogueira, R. J. M. C.; Araújo, F. P.; Melo, N. F.; Azevedo Neto, A. D. Physiological responses to salt stress in young umbu plants. Environmental and Experimental Botany, v.63, p.147-157, 2008.

Silva, M. T.; Amaral, J. A. B. Zoneamento risco climático para a cultura do amendoim no estado do Rio Grande do Norte. Revista de Biologia e Ciência da Terra, v.7, p.9399, 2007.
USDA - United States Department of Agriculture. Foreign Agricultural Service. http://www.fas.usda.gov/psdonline/ psdreport.aspx ?hidReport RetrievalName=BVS\&hidReport RetrievalID=918\&hidReportRetrievalTemplateID=1\#ancor. 14 Jun. 2010.

Tester, M.; Davenport, R.; Na+ tolerance and $\mathrm{Na}+$ transport in higher plants. Annals of Botany, v.91, n.5, p.503-527, 2003.

Zanandrea, L.; Nassi, F. L.; Turchetto, A. C.; Braga, E. J. B.; Peters, J. A.; Bacarin, M. A. Efeito da salinidade sob parâmetros de fluorescência em Phaseolus vulgaris. Revista Brasileira de Agrociência, v.12, p.157-161, 2006. 